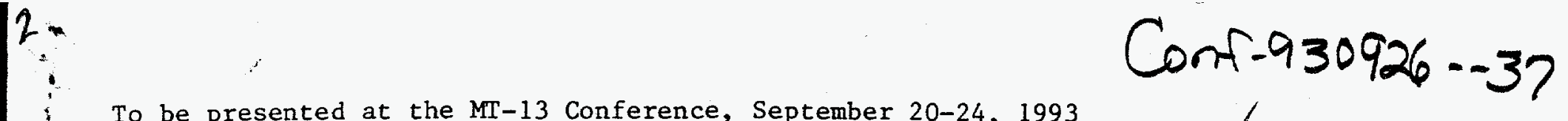

To be presented at the MT-13 Conference, September 20-24, 1993

Victoria Conference Centre Victoria, BC Canada BNL-49323

\title{
QUENCH PROPAGATION STUDY FOR FULL-LENGTH RHIC DIPOLE MAGNETS
}

J. Muratore, M. Anerella, G. Ganetis, M. Garber, A.K. Ghosh, A. Greene, R. Gupta, A. Jain, S. Kahn, E. Kelly, G. Morgan, A. Prodell, M. Rehak, E.P. Rohrer, W. Sampson, R. Shutt, R. Thomas, P. Thompson, P. Wanderer, E. Willen Brookhaven National Laboratory, Upton, New York 11973, U.S.A.'

\section{INTRODUCTION}

The study of quench propagation velocities and MIITs analysis of conductor hot spot temperatures during quench tests are important tools in the design, implementation, and verification of quench protection strategies in superconducting accelerator magnets. This type of investigation is facilitated by artificially inducing quenches with spot heaters mourited at carefully chosen locations on the magnet coils and which are closely bracketed by voltage taps. In this way, by the use of fast data acquisition systems, it is possible to measure the increase in voltage, and therefore resistance, of a short section of cable at the quench origin as the conductor temperature increases, and from this to determine the temperature. This is not possible in spontaneous quenches, where the quench origin is not known beforehand and therefore where there may be no voltage taps, as in production magnets. It is very straightforward, however, to measure the number of MIITs generated, since only the magnet coil current and voltage need be measured. The concept of MIITs then becomes a valuable diagnostic tool which can characterize the temperature behavior of a conductor during a quench and can be used to determine limits for safe operation of the magnet.

Research such as that described in this paper is therefore important in order to determine the validity of the MIITs approach and to establish a correlation between temperature and MIITs. In addition, the initial slope of the spot heater short section voltage signal or of the magnet coil voltage signal can be used to calculate the initial velocity.

Such quench velocity and temperature studies have been performed on various superconducting magnets for the RHIC collider project at Brookhaven National Laboratory in Upton, NY during the research phase of magnet development there. They were done as part of an extensive test program to evaluate the mechanical and magnetic performance of RHIC magnets under varying conditions. This paper presents the results for spot heater studies done on two of the full length RHIC prototype dipole magnets.

\section{MAGNET DESIGN AND CONSTRUCTION}

The two $9.7 \mathrm{~m}$-long, $8 \mathrm{~cm}$ aperture RHIC dipole magnets discussed in this paper are designated DRD009 and DRD010, and both share the same design and construction features.
Details of the design can be found in [1].

The conductor used in both magnets is a 30 strand cable manufactured by IGC Advanced Superconductors, Inc of Waterbury, CT and varies only slightly from nominal values of some parameters among the coil halves of the two magnets. Each strand has diameter $0.65 \mathrm{~mm}$ and consists of $6 \mu \mathrm{m} \mathrm{NbTi}$ filaments in a copper matrix. The copper RRR is nominally 70 after coil curing and the copper-to-superconductor ratio is nominally 2.18 . Theoretical quench current predicted from short sample cable measurements is about $6800 \mathrm{~A}$ at $4.3 \mathrm{~K}$ and spontaneous quench plateaus for both magnets reached that level or slightly higher.

\section{SPOT HEATER DESCRIPTION}

Each spot heater element is a $50.8 \mu \mathrm{m}$ thick rectangular strip. of stainless steel $38.1 \mathrm{~mm}$ long and $11.9 \mathrm{~mm}$ wide, mounted on the wide flat dimension of cable on a section of the midplane or pole turns. The mounting is accomplished by first stripping away a $50.8 \mathrm{~mm}$ length of cable insulation at the location where the heater is to be attached, then placing two layers of Kapton, the heater element strip, then another layer of Kapton. Also, there is a Kapton cap placed over the midplane of both the upper and lower coil halves, and this provides additional thickness of insulation between a midplane tum spot heater and the adjacent midplane turn of the other coil half. Projections on each end of the heater element strip are bent away from the cable and brought out into the leads, which are routed along the coil inner diameter to the outside of the magnet for connection to a pulser power supply. A quench is initiated by generating a pulse by capacitative discharge through the heating element with enough energy to heat the small section of conductor to its critical temperature. Energies needed to induce a quench depended on field strength, and therefore magnet current, at the quench location. For RHIC dipole magnets, $58 \mathrm{~ms}$ wide pulses were used, with amplitudes that ranged up to $11 \mathrm{~V}$, and corresponding energies up to about $4 \mathrm{~J}$.

For both magnets DRD009 and DRD010, two spot heaters were mounted on each of the upper and lower coil halves: spot heaters \#1 and \#3 were located $51.44 \mathrm{~cm}$ from the magnet lead end, on the pole turn of the lower and upper coil halves, respectively; spot heaters $\$ 2$ and $\# 4$ were $16.51 \mathrm{~cm}$ from the return end, on the midplane turn of the

'Work performed under contract with U.S. Department of Energy. 
lower and upper coils, respectively. Each spot heater was closely bracketed by voltage taps spaced $12.7 \mathrm{~cm}$ apart and equidistant from the spot heater center.

\section{EXPERIMENTAL PROCEDURES}

For a quench initiated by any of the spot beaters, conductor hot spot temperature could be measured by acquiring the voltage vs time signal for the short section of cable between the two bracketing voltage taps and dividing this by the current signal point-by-point to get the time development of the resistance as the section of cable, already normal, heats up. Temperature is then determined from a cemperature-resistance calibration curve obtained from measurements made on a short sample of the cable used in the magnet coil [2].

For each quench, MITTs values were calculated by integrating the square of the current signal waveform from the quench start time, determined by the onset of resistive voltage in the short section, to the time at which the current has decayed to zero. The relationship between MIITs and hot spot temperature is given by the expression

$$
\text { MIITS } \times 10^{6}-\int_{0}^{-} d t I^{2}(t)-S^{2} \int_{T_{0}}^{T_{\max }} d T\left(\frac{r}{1+r}\right) \frac{C(T)}{\rho(R R R, T, B)},(1)
$$

which is derived from a heat balance equation [3] that assumes adiabatic and constant field conditions, usually good assumptions during the initial part of the quench propagation. In this equation, $I$ is the magnet current, $T_{0}$ is the initial temperature, $T_{\max }$ is the hot spot temperature, $S$ is the conductor cross- section, $\mathrm{I}$ is the copper-to-superconductor ratio, $C$ is the specific heat per unit volume of conductor, $\rho$ is the copper resistivity, RRR is the copper residual resistivity ratio, and $B$ is the magnetic field at the quench spot.

As can be seen, if the conductor properties and the magnetic field at the quench location are known, the hot spot temperature can be calculated from MIITs by an explicit solution of (1). A computer model [4] which does this was used to calculate a theoretical hot spot temperature from the MIITs number for each of the quenches induced by the spot heaters in the study. Since only several quenches at most were done at each test current, and therefore field, it was not possible to fit the measured MIITs-temperature data points to theoretical correlation curves, each of which correspond to a selected field strength, as was done previously for other magnet test results [5]. However, by comparing temperatures predicted from the adiabatic model to the actual measured temperatures, it is possible to determine how closely measured data fit the model and its assumptions, and thus provide some insight into actual mechanisms occurring during quench propagation. In addition, it is also then possible to establish upper bounds for MIITs that give the safe conductor temperature limits of, typically, $500 \mathrm{~K}$. This is an important result, especially for production testing, since MIITs numbers can be easily determined but temperature cannot.

In addition to temperature and MIITs measurements, quench propagation velocities were also calculated from the voltage tap signal data and fitted to an adiabatic model [6] to obtain information on possible mechanisms at work during quenches. This model calculates theoretical initial quench velocities using a classical one-dimensional equation

$$
v_{\text {inis }}-A \sqrt{\frac{D P}{H}},
$$

where the parameters $D, P$, and $H$ are the equivalent longitudinal thermal diffusivity, the average power density per unit volume, and the enthalpy variation per unit volume, respectively, of the magnet coil. These parameters are fully discussed in [6]. The factor $A$ is actually a function which depends on several effects which are very difficult to measure, so that, in using this model, it is necessary to work backwards and scale the theoretical curve to fit the data and thus determine the value of the factor. These effects include cooling, the beat capacity of the insulation and helium, current sharing, and temperature dependence of the thermal conductivity and heat capacity of materials. This type of model has been used previously with some success [7].

The experimental initial quench velocity was calculated by measuring the slope $\mathrm{dV} / \mathrm{dt}$ of the resistive voltage increase with time and using the formula

$$
v_{\text {ini }}-\frac{S}{\rho(B) I} \frac{d V}{d t} \text {. }
$$

which can be derived from Ohm's Law and where $S$ is the cross-sectional area of the conductor, $I$ is the quench current, and the copper resistivity $\rho(B)$ is a known function of the magnetic field $B$.

For each magnet, the upper coil pole and midplane turn spot heaters were used to induce quenches at a series of magnet currents and the MIITs values were calculated. For each quench, the hot spot temperature was determined from measurements of resistance vs. time. Initial velocities were calculated from (3). These results were compared to theoretical calculations of temperature from MIITs and of initial quench velocities from the adiabatic, one-dimensional models discussed above. Results reported here were obtained at the nominal test temperature of $4.3 \mathrm{~K}$. The magnets were maintained at this temperature in the Horizontal Test Facility (Magcool) at Brookhaven by cooling with forced flow, supercritical helium gas.

\section{EXPERIMENTAL RESULTS}

\section{A. MIITs}

Figure 1 shows plots of MIITs vs $I_{q}$ for the upper coil spot heaters of both magnets. As can be seen, the pole turn quench data (Spot Heater \#3) agree very closely for both magnets, and the shapes of all plots are similar. Furthermore, they agree qualitatively with the results of studies done on other RHIC magnets and also on SSC magnets [5]. The 


\section{DISCLAIMER}

This report was prepared as an account of work sponsored by an agency of the United States Government. Neither the United States Government nor any agency thereof, nor any of their employees, make any warranty, express or implied, or assumes any legal liability or responsibility for the accuracy, completeness, or usefulness of any information, apparatus, product, or process disclosed, or represents that its use would not infringe privately owned rights. Reference herein to any specific commercial product, process, or service by trade name, trademark, manufacturer, or otherwise does not necessarily constitute or imply its endorsement, recommendation, or favoring by the United States Government or any agency thereof. The views and opinions of authors expressed herein do not necessarily state or reflect those of the United States Government or any agency thereof. 


\section{DISCLAIMER}

Portions of this document may be illegible in electronic image products. Images are produced from the best available original document. 
current for peak MIITs was about $58 \%$ of the spontaneous plateau quench current, and this behavior is also typical of other magnets and can be understood by a simple argument. The maximum MIITs will occur at some intermediate current at which the quench velocity has become fast enough to transport the heat away from the hot spot and limit the temperature there as the stored electromagnetic energy is converted to heat. This current is typically about $2 / 3$ of the quench plateau mean current and varies somewhat from magnet to magnet due to differences in conductor properties and cooling effects. The curves for the midplane turn quenches (Spot Heater \#4) fall below those for the pole turn probably because of increased azimuthal propagation to adjacent turns at the midplane. This effect has to be large enough to more than offset the effect of somewhat lower quench velocities in the midplane turn compared to the pole turn due to the lower field strength there.

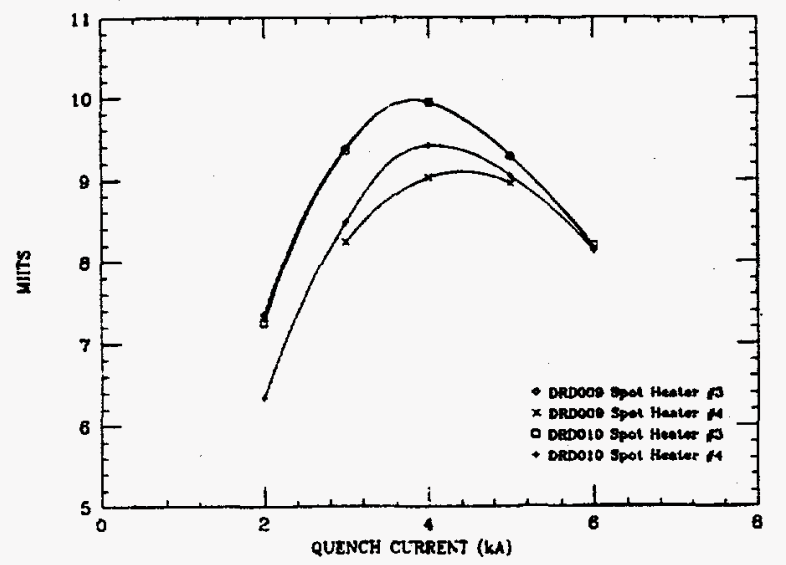

Figure 1. MIITs vs. quench current.

\section{B. Hot Spot Temperature}

Hot spot temperatures determined from resistance measurements made during the same quenches are plotted in Fig. 2. As can be seen, the pole turn data show good correlation of temperature with the MIITs numbers of Fig. 1, and both magnets again agree closely. The solid curve represents temperatures calculated for the measured MIITs of Fig. 1 with the adiabatic model (1) for the pole turn quenches. As can be seen, the theoretical curve approaches the data at the lower and higher fields but is as much as $100 \mathrm{~K}$ greater at about the peak MIITs point. This could imply that cooling from helium gas has a more non-negligible effect for quenches at intermediate fields. Other factors that may be causing this behavior include temperature dependence of thermal conductivity and specific heat of the various materials involved.

The dashed curve in Fig. 2 was calculated from (1) for the MIITs values measured during quenches in the midplane turn and does not show a good fit to the data above the peak MIITs point. MIITs and temperature do not seem to correlate well at the higher currents, as the hot spot temperatures do not drop off as quickly as they do for the pole turn quenches. In fact, the midplane temperatures actually surpass the corresponding pole turn temperatures in the higher current region. This behavior turns out to be consistent with the results of the velocity calculations in the next section, in which it is shown that the pole tum velocities diverge from the midplane velocities for the higher current region. This also may imply that cooling should not be neglected in this region as it is in the adiabatic theoretical curves.

It should be noted from the data in Fig.2 that the highest temperature experienced by the conductor at the hot spot was under 400K. Testing was done with a nominal quench detector threshold of $2 \mathrm{~V}$, so there is a significant quench



Figure 2. Hot spot temperature vs. quench current.

safety margin relative to the $500 \mathrm{~K}$ limit since the detector threshold may be set to a lower voltage and the delay between quench detection and power supply shutoff

decreased. This will result in even lower hot spot temperatures. It is evident that these magnets can operate safely with passive dump resistors and have no need for active devices such as strip heaters.

These results also show that MIITs-temperature correlation curves generated by (1) are still useful as upper bounds for MIITs values even though the data for midplane quenches at higher currents diverged from the temperature curve predicted from the measured MIITs by (1). At these higher currents, the temperatures are decreasing and do not pose a safety concern.

\section{Quench Velocity}

Initial quench velocities were calculated for all quenches by using (3) and are plotted in Figs. 3 and 4 for the pole turn and midplane turn, respectively. For both sets of data, theoretical initial velocity curves were calculated from (2) and included in the appropriate figures. In both cases, the scale factor A was set to 0.66 to obtain the best fit to the data. This implies that one or more of the physical mechanisms discussed above with respect to (2) is playing a significant 
role in the quench propagation process and cannot be ignored. The determination of the functional form of the factor $A$ with respect to parameters representing the individual mechanisms is difficult and beyond the scope of this work. However, it is clear that the one-dimensional model described by (2) does successfully represent the quench propagation process in a quantitative way. Note that the model-generated curves correctly predict the divergence of the pole turn velocity data from the midplane velocities as the quench current increases.

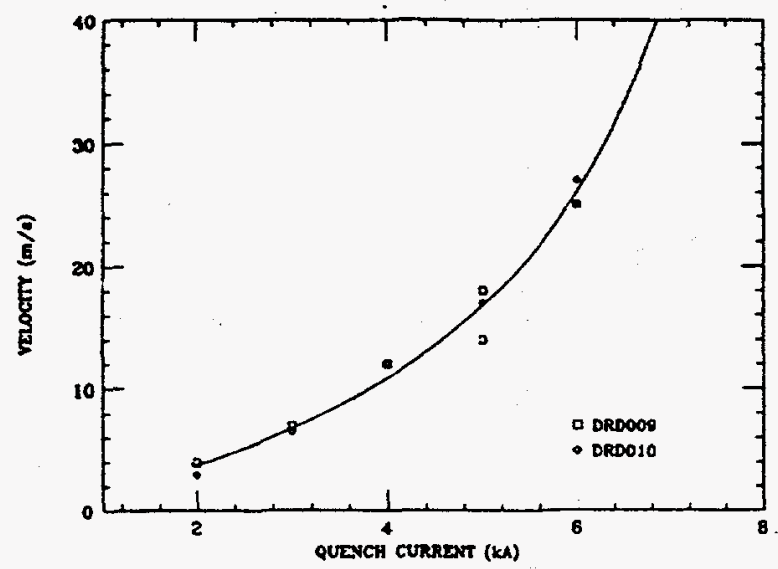

Figure 3. Initial quench velocities for pole turn. Solid curve is from model calculation.



Figure 4. Initial quench velocities for midplane turn. Solid curve is from model calculation.

This helps explain why the midplane quench temperatures decrease less quickly than the pole turn temperatures in the higher current region and even surpass them at some point.

In addition to the initial velocities, average velocities over the pole turn straight section were calculated by time-of-flight of the quench voltage signal as the quench front reached successive voltage taps. These data show a steeper increase of velocity with magnet current than the initial velocities in the pole turn. Qualitatively similar behavior has been measured in SSC dipole magnets [5] and is evidence that the quench front accelerates during propagation along the straight section of the coil.

\section{CONCLUSION}

During production testing of superconducting magnets, it is not possible to directly measure the conductor hot spot temperature during a quench due to the unavailability of voltage tap instrumentation, but it is straightforward to calculate MITTs numbers. The results reported in this work therefore show that correlation curves generated by the adiabatic model are still useful as upper bounds for MITTs values when temperatures cannot be measured. In order to get a more realistic fit to the data and be a better predictor of quench hot spot temperature, a model may need to account for various effects that may not be negligible during the initial phase of quench propagation, such as: 1) cooling by transfer of heat to the helium, 2) changes in the magnetic field at the quench location, 3) temperature dependence of the thermal conductivity and heat capacities of materials, and 4) current sharing effects. These conclusions are also consistent with the fit of quench velocity data to a one-dimensional adiabatic model. This study has also shown that RHIC dipole magnet coils are self-protecting without need for active quench protection devices such as strip heaters.

\section{ACKNOWLEDGMENT}

The authors would like to thank Phil Radusewicz of SSC Lab for his programming help and Arup Ghosh and Pat Thompson for helpful discussions.

\section{REFERENCES}

[1] Conceptual Design of the Relativistic Heavy Ion Collider, Brookhaven National Laboratory, May, 1989.

[2] M. Garber, Brookhaven National Laboratory, Upton, NY, private communication, 1992.

[3] N. M. Wilson, Superconducting Magnets, Oxford Clarendon Press, p. 200, 1983.

[4] A. Devred, MAGLIB Scientific Subroutine Library, Superconducting Supercollider Laboratory, 1992.

[5] J. Muratore et al, "Quench Propagation Study for BNL-Built, Full-Length, $50 \mathrm{~mm}$ Aperture SSC Model Dipoles," Supercollider 5, Plenum Press, 1993 (in press).

[6] A. Devred, "General Formulas for the Adiabatic Propagation Velocity of the Normal Zone, "IEEE Tran. Magnetics, 25 (2), 1698 (1989).

[7] A. K. Ghosh et al, "The Location of the Quench Origin in a Superconducting Accelerator Magnet," Proceedings of the IEEE Particle Accelerator Conf., p.1455, Washington, DC, 1987. 